\title{
PSA bounce, prognosis, and clues to the radiation response
}

\author{
Amar U. Kishan $\mathbb{D}^{1}$
}

Received: 16 April 2021 / Revised: 23 April 2021 / Accepted: 28 April 2021 / Published online: 18 May 2021

(c) The Author(s), under exclusive licence to Springer Nature Limited 2021

After definitive radiotherapy for prostate cancer, prostatespecific antigen (PSA) levels can transiently increase before ultimately dropping below the previous nadir sans intervention. First described in 1997 in the setting of low-doserate brachytherapy [1], this so-called "PSA bounce" phenomenon can lead to significant patient and provider anxiety out of concern for a recurrence. In fact, a significant impetus for developing the Phoenix definition for biochemical recurrence (BCR) - which defines a BCR as occurring at the time PSA exceeds a $2 \mathrm{ng} / \mathrm{mL}$ rise over the nadir PSA — was to avoid "false calls" of BCR based on the original American Society for Therapeutic Radiology and Oncology definition of three consecutive PSA rises with backdating to halfway between the time of nadir and the first rise [2, 3]. While various thresholds for defining a bounce exist, the most commonly used definition is a PSA rise of $\geq 0.2 \mathrm{ng} / \mathrm{mL}$ with a subsequent decrease equal to or below the value prior to the bounce [4]. Using this threshold, a recent meta-analysis of 50 reports estimated a pooled bounce frequency of $31 \%$, with heterogeneity across modalities and a generally higher frequency among younger patients and those with less aggressive disease [5].

Given the frequency of PSA bounces, a better understanding of their clinical relevance (beyond serving as anxiety-producing red herrings) is warranted. In this issue of Prostate Cancer and Prostatic Diseases, Urabe et al. report the results of a systematic review and meta-analysis evaluating the prognostic significance of PSA bounce [6]. The authors identified ten articles that compared BCR-free survival (BCRFS) outcomes between 2706 patients who experienced a PSA bounce and 6175 who did not. Most studies used a bounce threshold of $0.2 \mathrm{ng} / \mathrm{mL}$ and defined BCR using the Phoenix definition. Most patients (68.1\%)

Amar U. Kishan

aukishan@mednet.ucla.edu

1 Departments of Radiation Oncology and Urology, University of California, Los Angeles, CA, USA received low-dose-rate brachytherapy, with the second most common modality being conventionally fractionated radiotherapy (13.2\%). Due to limited heterogeneity, fixed-effect models were then used to calculate pooled hazard ratios (HRs). Only one included study also evaluated longer-term outcomes, and did report a similar association between bounce and distant metastasis-free survival, prostate cancerspecific survival, and overall survival; thus, the metaanalysis only focused on BCRFS [7]. Among the whole cohort, PSA bounce was associated with a decreased risk of BCR, with a pooled HR of 0.62 (95\% confidence interval [CI], 0.54-0.71). Hypothesizing a potential difference in prognostic effect base on radiotherapy modality, the authors reported a pooled HR of 0.38 (95\% CI, 0.27-0.55) among patients receiving low-dose-rate brachytherapy versus a pooled HR of 0.71 (95\% CI, 0.57-0.87) among patients receiving conventionally fractionated external beam radiotherapy.

Based on Urabe et al.'s meta-analysis, the occurrence of a bounce certainly seems to portend a favorable prognosis [6]. The analysis is not without limitations, which the authors acknowledge. All included studies were retrospective, with heterogeneous definitions for bounce and BCR. Studies that did not report HRs were not eligible for inclusion. And BCRFS, while of prognostic importance, is not necessarily a long-term clinical outcome.

Nonetheless, might a positive association between bounce incidence and prognosis have additional implications for understanding responses to radiation therapy? The pathophysiology underlying the bounce remains unknown, but emerging data support an inflammatory/immune etiology. A prospective study using three-dimensional magnetic resonance spectroscopic imaging in patients with an early rise in PSA after brachytherapy found that these patients had a diffuse pattern of increased metabolic activity (based on the [choline +creatine]:citrate ratio), while patients with a true recurrence had only focal increased activity [8]. In those with a benign increase in PSA, reduction in metabolic activity occurred as the PSA began to downtrend. In a cohort of 63 men treated with low-dose-rate brachytherapy, 
Yamamoto et al. observed a significantly higher CD8 and CD3 cell density in men who experienced a bounce [9]. Using mathematical modeling on the timing of the bounce, they postulated that radiotherapy initially kills radiosensitive infiltrating $\mathrm{T}$ cells, but, after a delay (corresponding to a decay in source activity for low-dose-rate brachytherapy), these cells return and launch an anti-tumor response that ultimate causes the bounce. They further postulated that anti-tumor immune action by these infiltrating lymphocytes is limited by the prostate primary tumor, and radiation-mediated tumor kill helps "unleash" these lymphocytes. Recent translational data from a trial of pre-operative stereotactic body radiotherapy showed a myeloid predominant cellular infiltrate two weeks after preoperative radiation, compared to a lymphoid predominant infiltrate without radiation [10]. These data would support the idea that initial radiation might kill tumor-infiltrating lymphocytes at a very early time point, though it cannot address if there is robust immune response at a later time point on the order of 6-12 months.

Though preliminary and unproven, an immune-mediated mechanism might explain the robust association of a boost with younger age and would be consistent with imaging evidence of diffuse metabolic activation during a bounce. This would also be consistent with a general trend of higher bounce frequencies when using radiation modalities that are thought to deliver more ablative doses of radiation, such as brachytherapy and stereotactic body radiotherapy, which are though to potentially stimulate a brisker immune response [5]. If the bounce does represent a stimulation of immune activity, this implies that a beneficial tumor-immune axis might be activated in prostate cancer via radiotherapy. This could in turn have implications for the role of radiation in priming an immune response in more advanced disease states. However, as yet, data on the prognostic utility of a bounce in more aggressive types of prostate cancer are unclear and, in fact, many reports seem to suggest that the incidence of a bounce is inversely associated with the aggressiveness of the disease [5].

Further studies will be needed to confirm whether the favorable prognostic association between BCRFS and bounce incidence can be leveraged to better understand the response to radiation for therapeutic benefit. Until then, the data presented by Udarbe et al. should be reassuring to physicians and the nearly $1 / 3$ rd of patients who have a PSA bounce after radiotherapy.

\section{Compliance with ethical standards}

Conflict of interest The author declares no competing interests.

Publisher's note Springer Nature remains neutral with regard to jurisdictional claims in published maps and institutional affiliations.

\section{References}

1. Wallner KE, Blasko J, Dattoli MJ. Evaluating cancer status. In: Wallner KE, Blasko J, Dattoli MJ, editors. Prostate brachytherapy made complicated. Seattle: SmartMedicine Press; 1997. p. 14:114.15.

2. Roach M 3rd, Hanks G, Thames H Jr, Schellhammer P, Shipley WU, Sokol GH, et al. Defining biochemical failure following radiotherapy with or without hormonal therapy in men with clinically localized prostate cancer: recommendations of the RTOG-ASTRO Phoenix Consensus Conference. Int J Radiat Oncol Biol Phys. 2006;65:965-74.

3. Pickles T. British Columbia Cancer Agency Prostate Cohort Outcomes InitiativeProstate-specific antigen (PSA) bounce and other fluctuations: which biochemical relapse definition is least prone to PSA false calls? An analysis of 2030 men treated for prostate cancer with external beam or brachytherapy with or without adjuvant androgen deprivation therapy. Int $\mathbf{J}$ Radiat Oncol Biol Phys. 2006;64:1355-9.

4. Caloglu M, Ciezki J. Prostate-specific antigen bounce after prostate brachytherapy: review of a confusing phenomenon. Urology. 2009;74:1183-90.

5. Darwis NDM, Oike T, Kubo N, Gondhowiardjo SA, Ohno TA-O. Characteristics of PSA Bounce after Radiotherapy for Prostate Cancer: A Meta-Analysis. LID - https://doi.org/10.3390/ca ncers 12082180. LID - 2180. (2072-6694 (Print)).

6. Urabe FK S, Tashihoro K, Kido M, Sasaki H, Aoki M, Kimura T, et al. Prognostic value of PSA bounce in prostate cancer following definitive radiation therapy: a systematic review and metaanalysis. Prostate Cancer Prostatic Dis. 2021.

7. Romesser PB, Pei X, Shi W, Zhang Z, Kollmeier M, McBride $\mathrm{SM}$, et al. Prostate-Specific Antigen (PSA) Bounce After DoseEscalated External Beam Radiation Therapy Is an Independent Predictor of PSA Recurrence, Metastasis, and Survival in Prostate Adenocarcinoma Patients. Int $\mathrm{J}$ Radiat Oncol Biol Phys. 2018;100:59-7.

8. Kirilova A, Damyanovich A Fau, Crook J, Crook J Fau, Jezioranski J, Jezioranski J Fau, et al. 3D MR-spectroscopic imaging assessment of metabolic activity in the prostate during the PSA "bounce" following 125iodine brachytherapy. Int J Radiat Oncol Biol Phys. 2011;79:371-8.

9. Yamamoto Y, Offord CP, Kimura G, Kuribayashi S, Takeda H, Tsuchiya $S$, et al. Tumour and immune cell dynamics explain the PSA bounce after prostate cancer brachytherapy. $\mathrm{Br} \mathrm{J}$ Cancer. 2016;115:195-202.

10. Nickols NG, Ganapathy E, Nguyen C, Kane N, Lin L, Diaz-Perez $\mathrm{S}$, et al. The intraprostatic immune environment after stereotactic body radiotherapy is dominated by myeloid cells. Prostate Cancer Prostatic Dis. 2021;24:135-9. 